\title{
Article \\ Anti-Periodontitis Effect of Ethanol Extracts of Alpinia Katsumadai Seeds
}

\author{
Seo Woo Shin and Young Sun Hwang *
}

check for updates

Citation: Shin, S.W.; Hwang, Y.S. Anti-Periodontitis Effect of Ethanol Extracts of Alpinia Katsumadai Seeds. Nutrients 2022, 14, 136. https:// doi.org/10.3390/nu14010136

Academic Editors: Sathish Kumar Natarajan, Jiujiu Yu and Corrine K Hanson

Received: 25 November 2021 Accepted: 25 December 2021 Published: 28 December 2021 Publisher's Note: MDPI stays neutral with regard to jurisdictional claims in published maps and institutional affiliations.

Copyright: (C) 2021 by the authors. Licensee MDPI, Basel, Switzerland. This article is an open access article distributed under the terms and conditions of the Creative Commons Attribution (CC BY) license (https:/ / creativecommons.org/licenses/by/ $4.0 /)$.

\author{
Department of Dental Hygiene, College of Health Science, Eulji University, 553, Sansung-Daero, Soojung-Gu, \\ Seongnam City 13135, Korea; kiteys@eulji.ac.kr \\ * Correspondence: peroxiredoxin@hanmail.net; Tel.: +82-31-740-7493
}

\begin{abstract}
Oral microbes are intimately associated with many oral and systemic diseases. Ongoing research is seeking to elucidate drugs that prevent and treat microbial diseases. Various functions of Alpinia Katsumadai seed extracts have been reported such as their anti-viral, anti-oxidant, anti-inflammatory, anti-puritic, anti-emetic, and cytoprotective effects. Here, we investigated the anti-periodontitis effect of an ethanol extract of Alpinia Katsumadai seeds (EEAKSs) on dental plaque bacteria (DPB)-induced inflammation and bone resorption. DPB and Porphyromonas gingivalis (P. gingivalis) were cultured and lipopolysaccharide (LPS) was extracted. Prostaglandin $\mathrm{E}_{2}\left(\mathrm{PGE}_{2}\right)$ and cyclooxygenase 2 (COX-2) levels were estimated using ELISA. Cytotoxicity was also verified. Proteases were screened using a protease antibody array method. Osteoclastic bone resorption was also investigated. EEAKSs suppressed $P$. gingivalis growth on agar plates. LPS prepared from dental plaque bacteria (DPB-LPS) and P. gingivalis (PG-LPS) significantly increased $\mathrm{PGE}_{2}$ and COX2 levels in immortalized gingival fibroblasts (IGFs), immortalized human oral keratinocytes (IHOKs), and RAW264.7 macrophage cells. However, DPB-LPS and PG-LPS-induced PGE 2 and COX-2 increases were effectively abolished by EEAKS treatment at non-cytotoxic concentrations. In the protease antibody array, matrix metalloproteinase (MMP)-2, MMP-3, MMP-7, kallikrein 10, cathepsin D, and cathepsin V levels were increased by PG-LPS stimulation. However, increases in protease levels except for cathepsin D were suppressed by EEAKS treatment. In addition, RANKL-induced osteoclast differentiation was significantly inhibited by EEAKS treatment, leading to reductions in resorption pit formation. These results suggest that EEAKSs exerted a beneficial oral health effect to help prevent DPB-mediated periodontal disease.
\end{abstract}

Keywords: ethanol extract of Alpinia Katsumadai seeds; periodontitis; dental plaque bacteria; lipopolysaccharide; inflammation; bone resorption

\section{Introduction}

Periodontal disease is a highly prevalent disease that occurs in over $90 \%$ of adults and is the main cause of tooth loss [1]. It is divided into gingivitis and periodontitis according to the severity of the disease. Gingivitis is a periodontal disease that can be quickly resolved and is limited to gum inflammation. However, periodontitis is an inflammatory lesion of periodontal tissue caused by proteolytic dental plaque bacteria present in the gingival margin. Therefore, a periodontal pocket is formed, gingival retraction occurs, and periodontal ligaments and alveolar bone are destroyed, causing tooth loss. Mixed infections with various bacteria present in dental plaque cause the inflammation of the periodontal tissue. In particular, Porphyromonas gingivalis ( $P$. gingivalis), which is present in oral biofilms and belongs to the red complex, has one of the highest risks for causing periodontal disease [2]. As inflammation progresses, P. gingivalis infiltrates into the gingival sulcular epithelium and is easily detected in the lesions of patients with periodontitis. Periodontal tissue is disrupted by metabolites, toxins, and proteolytic enzymes secreted by $P$. gingivalis in the process of attaching and penetrating epithelial cells and is also destroyed by cytokines produced by the host cells that react with $P$. gingivalis. 
Lipopolysaccharide, a structural component of the cell wall of Gram-negative bacteria, directly destroys tissues by acting as an endotoxin and stimulates the immune system to produce free radicals, prostaglandins, and various cytokines, causing inflammation [3]. These inflammatory mediators stimulate the secretion of collagenase from periodontopathogenic bacteria to decompose collagen in the periodontal tissues matrix, leading to the regression of the gums [4]. Therefore, suppressing the production of inflammatory mediators and proteolytic enzymes is very important for preventing periodontal disease. Recently, research on natural products for the prevention and treatment of periodontal disease has steadily progressed. Alpinia Katsumadai seeds are plants belonging to the ginger family. The peeled seeds are widely used as an anti-emetic medicine and to treat gastric disorders in oriental medicine [5]. The anti-oxidant, anti-viral, anti-asthmatic, and cytoprotective effects of Alpinia Katsumadai seeds have also been reported [5,6]. Ingredients such as diaryiheptanoids, flavonoids, monoterpenes, sesquiterpenoids, and stilbenes are known as the components of Alpinia Katsumadai seeds [7]. However, little is known about the intracellular events involved in the therapeutic effect of Alpinia Katsumadai seeds. In particular, the effect of Alpinia Katsumadai seeds on the inflammatory response is unknown.

In this study, we prepared an ethanol extract of Alpinia Katsumadai seeds (EEAKSs) and studied the effect of EEAKSs on periodontitis. For this, we verified the anti-bacterial effect of EEAKSs against dental plaque bacteria and $P$. gingivalis. The protective effect of EEAKSs on inflammation and osteoclastic bone resorption induced by dental plaque bacteria-fLPS (DPB-LPS) or P. gingivalis-LPS (PG-LPS) was also investigated. This result suggests that EEAKSs can be an effective agent in the prevention and treatment of periodontitis.

\section{Materials and Methods}

\subsection{Cell Lines and Culture Media}

Immortalized human gingival fibroblasts (IGFs) and immortalized human oral keratinocytes (IHOKs) were kindly provided by the Yonsei University College of Dentistry, Oral Cancer Institute (Seoul, Korea) and cultured in Dulbecco's modified Eagle's medium (DMEM)/F-12 medium (3:1 ratio) with 10\% fetal bovine serum as previous detailed [8]. RAW264.7 macrophage cells were cultured in RPMI 1640 containing 10\% fetal bovine serum (Gibco BRL).

\subsection{Bacterial Culture}

The Pophyromonas gingivalis strain ATCC 33277 was purchased from ATCC (Manassas, VA, USA) and cultured in Wilkins-Chalgren anaerobe broth (KisanBio, Seoul, Korea) and on Wilkins-Chalgren agar (KisanBio, Seoul, Korea) at $37{ }^{\circ} \mathrm{C}$ in an anaerobic incubator $(80 \%$ $\mathrm{N}_{2}, 10 \% \mathrm{CO}_{2}$, and $10 \% \mathrm{H}_{2}$ gas mix). Aliquots of $50 \mu \mathrm{L}$ bacterial culture were inoculated into $5 \mathrm{~mL}$ of medium under anaerobic conditions and incubated overnight before experiments. An anaerobic chamber (Modular incubator chamber, MIC-101, Billups-Rothenberg Inc., Shirley, NY, USA) was used to create anaerobic environment. An optical density of the bacterial culture was approximately $0.8(600 \mathrm{~nm})$. For agar plate cultures, a bacterial suspension was inoculated onto the top agar and bacterial growth was assessed. Dental plaque bacteria from dental plaques were cultured in brain-heart infusion (BHI) broth (Becton, Dickinson and Company, Baltimore, MD, USA) at $37^{\circ} \mathrm{C}$. Dental plaques were obtained by a dental hygienist through regular scaling from a participant. Informed consent was given by the participant.

\subsection{Ethanol Extracts of Alpinia Katsumadai Seeds}

The ethanol extracts of Alpinia Katsumadai seeds (EEAKSs) was provided by COSMAX Inc. R\&I Center (Seongnam, Korea). Alpinia Katsumadai seeds were ground to a fine powder and extracted material in $70 \%$ ethanol. A voucher specimen was deposited at the by COSMAX Inc. R\&I Center (Seongnam, Korea). 


\subsection{Lipopolysaccharide Extractions from Dental Plaque Bacteria}

An LPS extraction kit (iNtRON Biotechnology, Seongnam, Korea) was used for lipopolysaccharides (LPSs) extraction from dental plaque bacteria according to the manufacturer's manuals. The Limulus Amebocyte Lysate Chromogenic Endotoxin Quantitation Kit (Pierce Biotechnology, Rockford, IL, USA) was used for LPS quantification. Escherichia coli O111:B4 was used as the concentration standard. Ten endotoxin units (EU)/mL equaled approximately $1 \mathrm{ng} / \mathrm{mL}$.

\subsection{Enzyme-Linked Immunosorbent Assay}

The level of human Prostaglandin $\mathrm{E}_{2}\left(\mathrm{PGE}_{2}\right)$ and human cyclooxygenase-2 (COX2) was estimated by an ELISA kit. The following ELISA kits were purchased from their respective sources: human PGE $_{2}$ (KGE004B; R\&D Systems, Minneapolis, MN, USA), human COX-2 (ab267646; Abcam). Moreover, $1 \times 10^{5}$ cells were cultured in media with DPB-LPS and/or EEAKSs for $24 \mathrm{~h}$. The medium was used for analysis.

\subsection{Cytotoxicity Assay}

Cytotoxicity was tested with 3-(4,5dimethylthiazol-2-yl)-2,5-diphenyltetrazolium bromide (MTT) assay (Sigma-Aldrich, St Louis, MO, USA). Briefly, $1 \times 10^{4}$ cells was cultured with complete medium with or without EEAKSs for $24 \mathrm{~h}$. Cell medium was replaced with medium containing $5 \mathrm{mg} / \mathrm{mL}$ MTT and further cultured for $2 \mathrm{~h}$. Moreover, $100 \mu \mathrm{L}$ dimethyl sulfoxide (DMSO) was added to dissolve the purple product. The absorbance was measured at $570 \mathrm{~nm}$ using a microplate reader (SynergyTM HTX Multi-Mode Microplate Reader; BioTek Instruments Inc., Winooski, VT, USA).

\subsection{Protease Antibody Array}

The Proteome Profiler Human Protease Array Kit (R\&D Systems Inc., Minneapolis, MN, USA) was used for the antibody array. Among the confluence cells, $70 \%$ were cultured in serum-free medium containing DPB-LPS and/or EEAKSs for $24 \mathrm{~h}$. The culture medium was harvested by centrifugation at $1000 \mathrm{rpm}$ for $5 \mathrm{~min}$ and was used for conditioned medium (CM). Protease antibody array membrane was incubated with $\mathrm{CM}$ overnight and the following procedure was performed according to the manufacturer's protocols. Relative enhanced chemiluminescence (ECL) signal levels pf proteases were compared using Image J program (National Institutes of Health, Bethesda, MA, USA).

\subsection{Osteoclast Formation}

Mouse bone marrow-derived macrophages (BMMs) were prepared from the tibias of 4-week-old ICR mice using Histopaque and cultured in minimum essential medium alpha medium ( $\alpha$-MEM) containing 10\% FBS and M-CSF (30 ng/mL; R\&D System, Minneapolis, $\mathrm{MN}, \mathrm{USA})$. Moreover, $5 \times 10^{4}$ mouse BMMs cells were cultured in M-CSF $(30 \mathrm{ng} / \mathrm{mL})$, recombinant mouse soluble RANK ligand (sRANKL) $(100 \mathrm{ng} / \mathrm{mL}$; Koma Biotech, Seoul, Korea), DPB-LPS $(1 \mu \mathrm{g} / \mathrm{mL})$, and/or EEAKSs $(10 \mu \mathrm{g} / \mathrm{mL})$ for 10 days with replacement with fresh medium. After fixation, cells were reacted with the acid phosphatase, leukocyte (TRAP) kit (Sigma-Aldrich, St. Louis, MO, USA) according to the manufacturer's manuals. TRAP-positive multinucleated cells ( $\geq 3$ nuclei) were imaged and counted.

\subsection{Pit Formation}

Osteo assay surface polystyrene stripwells (Corning, Corning, NY, USA) were used to observe the pit formation by functional osteoclast. The $5 \times 10^{4}$ mouse BMMs cells prepared from the tibias of ICR mice were cultured in $\alpha$-MEM media containing M-CSF ( $30 \mathrm{ng} / \mathrm{mL}$ ), sRANKL $(100 \mathrm{ng} / \mathrm{mL})$, DPB-LPS $(1 \mu \mathrm{g} / \mathrm{mL})$, and/or EEATSs $(10 \mu \mathrm{g} / \mathrm{mL})$ for 2 weeks. Culture medium was replaced with fresh medium. Sodium hypochlorite solution was then added to the remaining lysis cells. The resorption pit was washed with PBS and images were captured. 


\subsection{Statistical Analysis}

InStat GraphPad Prism ver. 5.01 statistical software (GraphPad Software, Inc., San Diego, CA, USA) was used for statistical analyses. Non-parametric Kruskal-Wallis tests with Dunn's post hoc analysis was employed for multiple comparisons. The data are expressed as the mean \pm standard error of the mean (SEM). $p<0.05$ was considered statistically significant.

\section{Results}

\subsection{Effects of EEAKSS on P. gingivalis Growth}

Porphyromonas gingivalis is a major pathogenic bacterium of chronic periodontitis [9]. To test the effect of EEAKSs on $P$. gingivalis growth, cells were cultured until the optical density was approximately $0.8(600 \mathrm{~nm})$ and $10 \mu \mathrm{L}$ of bacterial solution was inoculated onto an agar plate, and cultured with various concentrations of EEAKSs for $48 \mathrm{~h}$. As shown in Figure 1, EEAKSs effectively suppressed $P$. gingivalis growth. As the concentration of the EEAKSs treatment increased, the number of colonies significantly decreased.

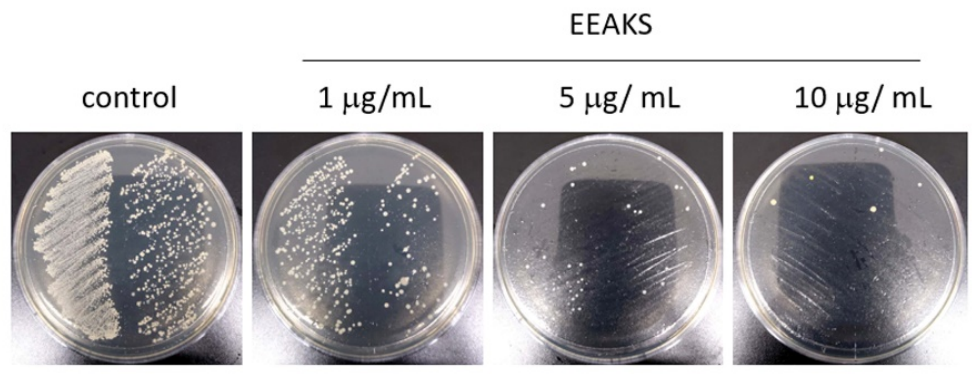

Figure 1. EEAKSs suppress Porphyromonas gingivalis growth. The effect of EEAKSs on $P$. gingivalis growth was investigated on Wilkins-Chalgren agar plates. P. gingivalis were cultured until optical density was approximately $0.8(600 \mathrm{~nm})$ and $10 \mu \mathrm{L}$ of bacterial solution was cultured on an agar plate with or without EEAKSs for $48 \mathrm{~h}$ in anaerobic chamber. Representative images are shown.

\subsection{Effect of EEAKSs on Bacterial LPS-Induced PGE 2 and COX-2 Levels}

The synthesis of $\mathrm{PGE}_{2}$ begins with the production of arachidonic acid from membrane phospholipids by the enzymatic reaction of phospholipase $A_{2}$, which is catalyzed by cyclooxygenase (COX). COX-2 is involved in both acute and chronic inflammatory response by producing $\mathrm{PGE}_{2}$ [10]. An increased $\mathrm{PGE}_{2}$ level was observed in gingival crevicular fluids of patients with periodontal disease [11]. To observe the effect of EEAKSs on inflammation, we first prepared bacteria (DPB) from dental plaques through regular scaling $(n=3)$, and the LPS of DBP (DPB-LPS) was extracted. LPS from P. gingivalis (PG-LPS) was also prepared by the same extraction protocol. Then, we tested the effect of DPB-LPS and PG-LPS on the $\mathrm{PGE}_{2}$ levels of various types of human cell lines. Moreover, $1 \mu \mathrm{g} / \mathrm{mL}$ of each DPB-LPS-1, DPB-LPS-2, DPB-LPS-3, or PG-LPS was treated to media for $24 \mathrm{~h}$ and ELISA analysis was performed. As shown in Figure 2A, a significant increase in PGE $_{2}$ levels by DPB-LPS was observed in the IGF, IHOK, and RAW264.7 cells. COX-2 levels were also increased by stimulation with $1 \mu \mathrm{g} / \mathrm{mL}$ DPB-LPS-1, DPB-LPS-2, DPB-LPS-3, or PG-LPS. The increases in $\mathrm{PGE}_{2}$ and COX-2 levels by DPB-LPSs or PG-LPS were different in function of the cell line. DPB-LPS-2 induced the most effective inflammatory response and was used for further studies (DPB-LPS). Then, the cells were treated with EEAKSs and the levels of $\mathrm{PGE}_{2}$ and COX-2 were analyzed. As shown in Figure 2B, the $\mathrm{PGE}_{2}$ and COX-2 levels increased by DPB-LPS or PG-LPS were significantly decreased by the $10 \mu \mathrm{g} / \mathrm{mL}$ EEAKS treatment in the various types of cells. To investigate the effect of EEAKSs on the viability of IGF, IHOK, and RAW264.7 cells, the MTT assay was performed. No apparent cytotoxicity in IGF, IHOK, and RAW264.7 cells was observed at $\leq 20 \mu \mathrm{g} / \mathrm{mL}$ EEAKSs, but weak cell growth inhibition was observed at $\geq 25 \mu \mathrm{g} / \mathrm{mL}$ EEAKSs (Figure 2C). Thus, EEAKSs not only inhibited the growth of dental plaque bacteria and $P$. gingivalis through its anti-bacterial effect but 
also inflammation by suppressing bacterial-induced $\mathrm{PGE}_{2}$ and COX-2 expression, thereby providing a beneficial effect in the prevention and management of periodontitis.

(A)
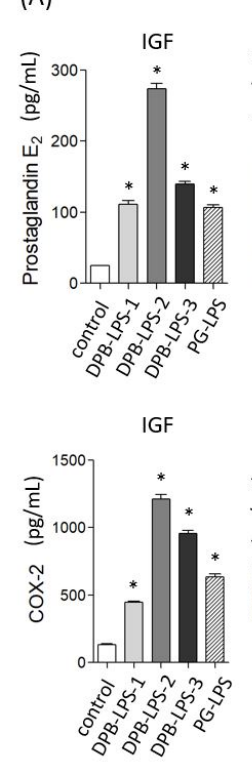
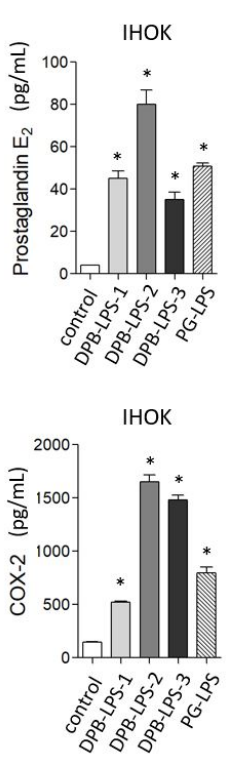
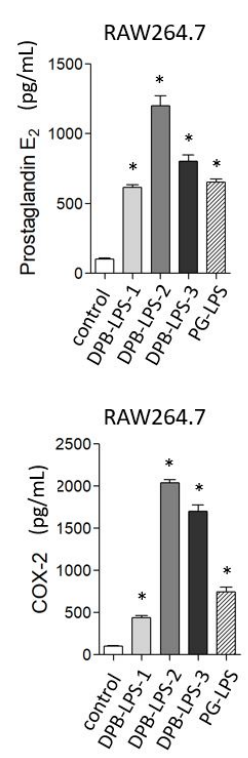

(B)
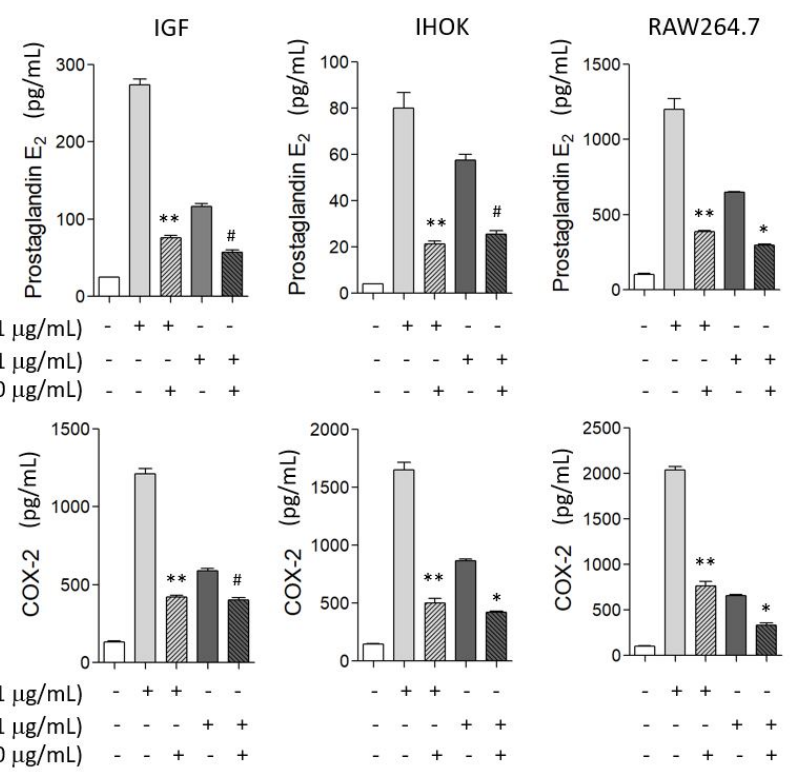

(C)
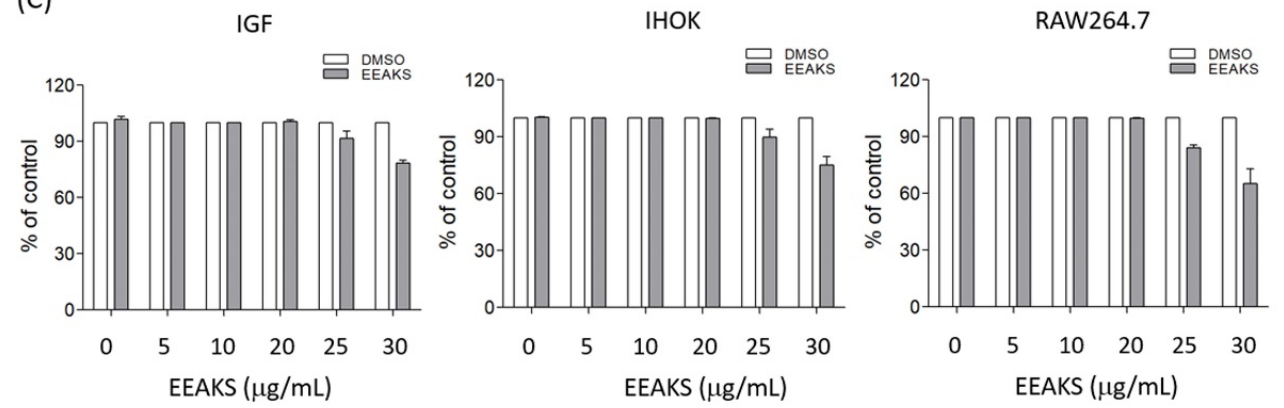

Figure 2. Effect of the EEAKSs on dental plaque bacterial-LPS-induced prostaglandin $\mathrm{E}_{2}$ and cyclooxygenase-2. (A) The effect of bacterial LPS on PGE $_{2}$ and COX-2 levels in immortalized gingival fibroblast (IGF), immortalized human oral keratinocyte (IHOK), and RAW264.7 macrophage. Three DPB-LPS (DPB-LPS-1, DPB-LPS-2, DPB-LPS-3) were prepared from dental plaques and each DPB-LPS $(1 \mu \mathrm{g} / \mathrm{mL})$ was treated to media for $24 \mathrm{~h}$ and $\mathrm{PGE}_{2}$ and COX-ELISA analysis was performed. PG-LPS was also extracted from $P$. gingivalis. Moreover, $1 \mu \mathrm{g} / \mathrm{mL}$ PG-LPS was treated to media for $24 \mathrm{~h}$ and ELISA analysis was also performed. The data are expressed as the mean \pm standard error of the mean (SEM). ${ }^{\#} p<0.01,{ }^{*} p<0.001$ vs. without bacterial LPS control medium from each cell line. (B) The effect of EEAKSs on bacterial LPS-induced PGE 2 and COX-2 expression. EEAKSs $(10 \mu \mathrm{g} / \mathrm{mL})$ were treated in the media with DPB-LPS or PG-LPS $(1 \mu \mathrm{g} / \mathrm{mL})$ and the PGE 2 and COX-2 level was estimated using the ELISA kit. ${ }^{* *} p<0.001$ vs. only DPB-LPS treated cells, ${ }^{*} p<0.05,{ }^{*} p<0.01$ vs. only PG-LPS treated cells. (+), add; (-), no add. (C) The effect of EEAKSs on human cell growth. Immortalized human oral keratinocytes (IHOKs), immortalized human gingival fibroblasts (IGFs), and RAW264.7 macrophage cells were treated with indicated concentration of EEAKSs for $24 \mathrm{~h}$ and MTT assay was performed. DMSO was treated as the assay control.

\subsection{Identify the Proteases Involved in PG-LPS-Induced Inflammation}

Inflammation is a defense mechanism to protect tissues from infection or injury, and proteolytic activity is induced to remove and recover damaged tissues. However, proteases secreted by bacteria in the periodontal pocket cause periodontitis by destroying the surrounding tissues and causing alveolar bone resorption [12]. To elucidate the proteases involved in $P$. gingivalis-induced inflammation, IHOK cells were treated with $1 \mu \mathrm{g} / \mathrm{mL}$ 
PG-LPS with or without $10 \mu \mathrm{g} / \mathrm{mL}$ EEAKSs and the protease antibody array assay was performed using the culture medium. In only the PG-LPS-treated IHOK cells, the proteases profiles were increased for matrix metalloproteinase (MMP)-7 (1), Kallikrein 10 (2)), Cathepsin V (3), Cathepsin D (4), MMP-2 (5), and MMP-3 (6) by 1.6 2.5-fold compared to the dimethyl sulfoxide (DMSO) control (Figure 3). However, the increased protease levels were significantly decreased by the EEAKS treatment-except for cathepsin D.
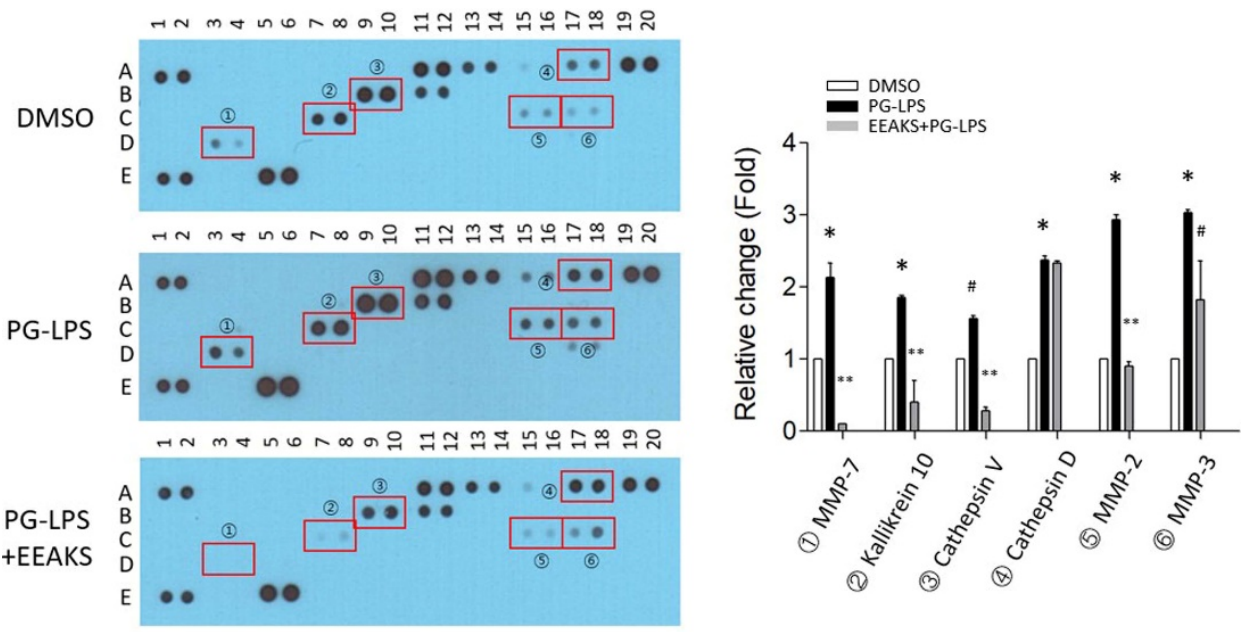

Figure 3. Effects of EEAKSs on PG-LPS-induced proteases levels. PG-LPS $(1 \mu \mathrm{g} / \mathrm{mL})$ treated in IHOK cell culture medium with or without EEAKSs $(10 \mu \mathrm{g} / \mathrm{mL})$ and culture medium $(\mathrm{CM})$ was applied onto protease antibody array membrane. DMSO was treated as the control. Altered factors are indicated in the images as red rectangles and circled numbers. The list altered factor is shown as fold-change in the graph. ${ }^{*} p<0.01$ vs. DMSO-treated medium, ${ }^{* *} p<0.001$ vs. PG-LPS-treated medium, ${ }^{\#} p<0.05$ vs. PG-LPS-treated medium.

\subsection{Effects of EEAKSs on RANKL-Induced Osteoclast Formation and Bone Resorption}

Alveolar bone loss is a hallmark of periodontitis progression [13]. To observe the effect of EEAKSs on osteoclastic bone resorption, bone marrow-derived macrophage (BMM) cells were treated with RANKL with or without EEAKSs and osteoclast differentiation was observed. TRAP-positive multinucleated osteoclasts were clearly detected in the medium containing $100 \mathrm{ng} / \mathrm{mL}$ of RANKL (Figure 4). However, EEAKSs significantly inhibited RANKL-induced osteoclast formation in a dose-dependent manner. Significant reductions in osteoclast numbers were observed by treatment with $\geq 5 \mathrm{mg} / \mathrm{mL}$ EEAKSs. The bone resorption activity of mature osteoclasts was also measured by the pit formation assay. Consistent with the osteoclast formation studies, resorption pits induced by RANKL were significantly inhibited by EEAKS treatment. These results indicate that EEAKSs inhibited RANKL-induced osteoclast formation and bone resorption. 


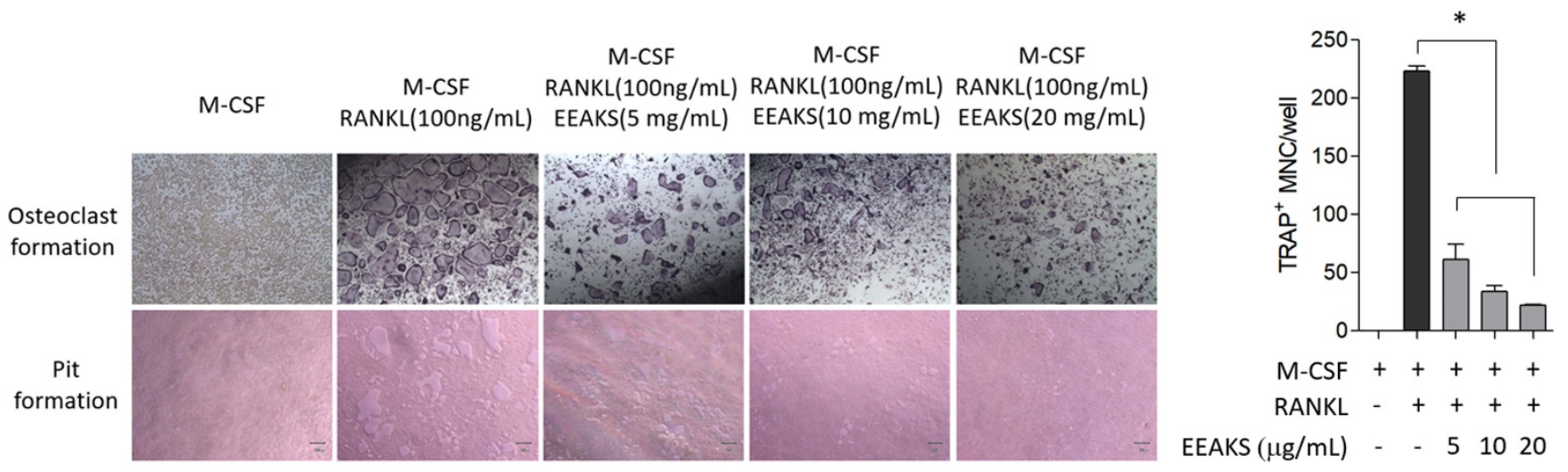

Figure 4. Effect of EEAKSs on RANKL-induced osteoclastic bone resorption. Mouse BMM cells were treated with M-CSF (30 ng/mL) and RANKL $(100 \mathrm{ng} / \mathrm{mL})$ with or without EEAKSs $(5$ or $10 \mu \mathrm{g} / \mathrm{mL}$ ) and were stained to detect the expression of TRAP. Resorptive pit was also detected on calcium phosphate apatite-coated plates under light microscopy. The total number of TRAP-positive multinucleated ( $\geq 3$ nuclei) osteoclasts (MNCs) per well is graphically presented. The data are expressed as the mean \pm standard error of the mean (SEM). ${ }^{*} p<0.001$ vs. M-CSF+RANKL condition. $(+)$, add; (-), no add.

\section{Discussion}

Periodontitis is a highly prevalent periodontal disease found in $20-50 \%$ of the global population [14]. In the Republic of Korea, gingivitis and periodontal disease have remained the second most frequent diseases over the past decade. In particular, as the prevalence of periodontal disease increases with age, periodontal disease is becoming an obstacle in nutritional management to maintain individual health and prevent diseases. Tooth loss is strongly associated with malnutrition in older adults [15]. Therefore, research has been conducted to derive effective ingredients for the prevention and treatment of periodontal disease, and many natural ingredients have been suggested. Herbal extracts, catechin, theaflavin, curcumin, and garlic components were reported to be effective ingredients for periodontitis. In gingival cells, herbal extract, theaflavin, and curcumin inhibited $\mathrm{PGE}_{2}$, IL-6, IL-8, and tumor necrosis factor (TNF)-a levels [16-20]. In animal studies, sumac extract, epicatechin gallate, epigallocatechin gallate, and curcumin derivative inhibited the alveolar bone resorption [21-24]. Garlic ingredients such as allicin, diallyl sulfide, and aged garlic extract also inhibited the growth of periodontitis-causing bacteria such as P. gingivalis, A. actinomycetemcomitans, and F. nucleatum [25-28].

Although Alpinia katsumadai seeds have various effects such as anti-oxidant and antiviral, their role in inflammatory response by endogenous oral bacteria has not been reported. In this study, we sought to confirm the effect of Alpinia katsumadai seed extracts on the inflammatory response caused by periodontopathic bacteria. In addition, we also tried to confirm the effect of Alpinia katsumadai seed extracts on alveolar bone resorption observed in aggressive periodontitis. To test its anti-bacterial activity, $P$. gingivalis was cultured on agar plates containing different concentrations of EEAKSs. P. gingivalis growth was significantly inhibited by EEAKSs in a dose-dependent manner. This result encouraged us to study the role of EEAKSs in the inflammatory response and alveolar bone resorption observed in periodontitis. For these studies, dental plaque bacteria (DPB) were cultured from dental plaques through regular scaling, and whether DPB induced inflammation in various human cell lines was analyzed. However, in vitro co-cultures of human cell lines and DPB caused $\mathrm{pH}$ changes in culture medium during overnight incubation. LPS was therefore extracted from DPBs (DPB-LPS), and its capacity to induce inflammation was verified. LPS from $P$. gingivalis (PG-LPS) was also prepared by the same extraction procedure. LPS extracted from bacteria was quantified using the Endotoxin Quantitation Kit for further studies. In immortalized gingival fibroblasts (IGFs), immortalized human oral keratinocytes (IHOKs), and RAW 264.7 macrophages, $1 \mu \mathrm{g} / \mathrm{mL}$ of DPB-LPS and PG-LPS significantly increased 
the $\mathrm{PGE}_{2}$ and COX-2 levels. There was a difference in the stimulation degree according to the three extracted LPSs, but strong PGE 2 and COX-2 stimulation by DPB-LPS or PG-LPS was observed compared to the controls. However, the $\mathrm{PGE}_{2}$ and COX-2 secretion increased by DPB-LPS or PG-LPS was significantly decreased by EEAKS treatment and the inhibitory effect was dose-dependent. The concentrations of EEAKSs $(5-10 \mu \mathrm{g} / \mathrm{mL})$ that inhibited bacterial growth and $\mathrm{PGE}_{2}$ and COX-2 secretion did not induce cytotoxicity in IGFs, IHOKs, and RAW 264.7 cells. These results suggest that EEAKSs are a useful agent for inhibiting periodontitis-causing bacterial growth without cytotoxicity.

In periodontitis, many types of protease secretion are increased and play roles in matrix destruction and alveolar bone regression [29]. To identify the proteases induced by PG-LPS, culture media from IHOK cells treated with PG-LPS and/or EEAKSs for $24 \mathrm{~h}$ were analyzed using the human protease antibody array kit in duplicate with 34 different protease antibodies. Among the proteases increased by PG-LPS, MMP-2, MMP-3, MMP-7, kallikrein 10, and cathepsin V levels were decreased by EEAKS treatment. MMPs are proteolytic enzymes involved in the degradation of the extracellular matrix of various tissues including bones [30]. Kallikrein is involved in bone resorption by the kallikreinkinin system and the coagulation cascade [31]. Cathepsins play roles in lysosomal protein turnover, which contributes to a plethora of physiological processes, such as antigen presentation, bone remodeling, and epidermal homeostasis [32]. The induction of MMP-2 and MMP-3 in osteoblasts is essential for bone resorption [33]. Additionally, MMP-7 promotes prostate cancer-induced osteolysis via the solubilization of the receptor activator of nuclear factor-kappaB (RANKL) [34]. Although the protein level of kallikrein 10 may change during disease initiation and progression, kallikrein 10 overexpression is correlated with breast cancer aggressiveness [35]. Cathepsin V has potent elastolytic activity and accelerates the destruction of the elastin matrix in diseased arteries [36]. Osteoclast differentiation and bone resorption activity require stimulation by RANKL expressed on osteoblasts [37]. In this study, we also observed the role of EEAKSs in osteoclastogenesis. In the RANKL-induced osteoclast differentiation process, EEAKS treatment significantly inhibited osteoclast formation in a dose-dependent manner. Taken together, the bone resorption activity of mature osteoclasts was also suppressed by EEAKS treatment. These results suggest that EEAKSs could be an effective ingredient in controlling proteases secretion and alveolar bone regression caused by periodontitis. In this study, the antibacterial and anti-inflammatory activity of EEAKSs against anaerobes was confirmed using the anaerobic P. gingivalis (Figure 5). However, in the case of dental plaque bacteria, these activities were only observed for bacteria cultured under aerobic conditions. It is a limitation of our study that antibacterial and anti-inflammatory activities were not observed against anaerobic bacteria in dental plaque. In addition, if analytical grade EEAKSs are available, a further study to compare the activity using them as an experimental control will be needed.

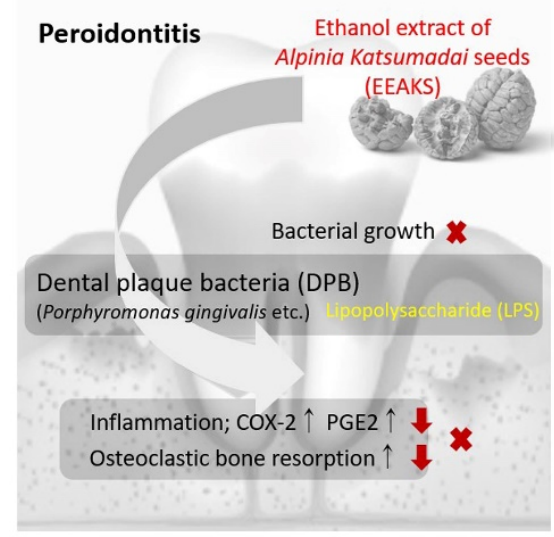

Figure 5. The proposed mechanism of ethanol extract of Alpinia Katsumadai seeds' (EEAKSs') antiperiodontitis effects. 


\section{Conclusions}

Periodontitis is an aggressive bacterial inflammatory disease and $P$. gingivalis is the representative causative bacterium. EEAKSs effectively inhibit $P$. gingivalis growth and abolish bacterial LPS-induced proteases levels. In addition, EEAKSs are an effective plant ingredient that can regulate the expression of inflammatory factors caused by bacterial LPS. Therefore, EEAKSs can be suggested as an antibacterial and anti-inflammatory agent for preventing and controlling periodontitis. Further studies are needed to isolate the functional components of the extract and to determine its delicate activity for pharmaceutical use.

Author Contributions: Conceptualization, S.W.S. and Y.S.H.; data curation, S.W.S. and Y.S.H.; formal analysis, S.W.S. and Y.S.H.; methodology, S.W.S. and Y.S.H.; project administration, Y.S.H.; software, S.W.S. and Y.S.H.; writing—original draft, Y.S.H.; writing—review and editing, S.W.S. and Y.S.H. All authors have read and agreed to the published version of the manuscript.

Funding: This research was supported by Basic Science Research Program through the National Research Foundation of Korea (NRF) funded by the Ministry of Education, Science and Technology (2018R1D1A1B07042035) and by Eulji University in 2021.

Institutional Review Board Statement: Approval was given by the Institutional Review Board of Eulji University (EU19-62). Informed consent for the collection of dental plaque was given by individual participators.

Informed Consent Statement: Informed consent was obtained from all subjects involved in the study.

Data Availability Statement: The data and materials of this article are included within the article.

Conflicts of Interest: The authors declare that there is no conflict of interest.

\section{Abbreviations}

A. Katsumadai, Alpinia Katsumadai seed; LPS, Lipopolysaccharide; COX-2, Cyclooxygenase-2; $\mathrm{PGE}_{2}$, Prostaglandin E2; RANKL, Receptor activator of nuclear factor $\mathrm{\kappa B}$ ligand.

\section{References}

1. Könönen, E.; Gursoy, M.; Gursoy, U.K. Periodontitis: A Multifaceted Disease of Tooth-Supporting Tissues. J. Clin. Med. 2019, 8, 1135. [CrossRef]

2. Suzuki, N.; Yoneda, M.; Hirofuji, T. Mixed Red-Complex Bacterial Infection in Periodontitis. Int. J. Dent. 2013, $2013,587279$. [CrossRef]

3. Sampath, V. Bacterial endotoxin-lipopolysaccharide; structure, function and its role in immunity in vertebrates and invertebrates. Agric. Nat. Resour. 2018, 52, 115-120. [CrossRef]

4. Barry, M.; Stephen, W.C. Proteolytic and hydrolytic enzymes from putative periodontal pathogens: Characterization, molecular genetics, effects on host defenses and tissues and detection in gingival crevice fluid. Periodontology 2003, 31, 105-124.

5. Kim, H.H.; Kwon, H.J.; Ryu, Y.B.; Chang, J.S.; Cho, K.O.; Hosmillo, M.D.; Rho, M.C.; Park, S.J.; Lee, W.S. Antiviral activity of Alpinia katsumadai extracts against rotaviruses. Res. Vet. Sci. 2012, 92, 320-323. [CrossRef]

6. $\quad$ Lee, M.Y.; Lee, N.H.; Seo, C.S.; Lee, J.A.; Jung, D.; Kim, J.H.; Shin, H.K. Alpinia katsumadai seed extract attenuate oxidative stress and asthmatic activity in a mouse model of allergic asthma. Food Chem. Toxicol. 2010, 48, 1746-1752. [CrossRef]

7. Nam, J.-W.; Seo, E.-K. Structural Characterization and Biological Effects of Constituents of the Seeds of Alpinia katsumadai (Alpina Katsumadai Seed). Nat. Prod. Commun. 2012, 7, 795-798. [CrossRef] [PubMed]

8. Hwang, Y.S.; Chung, W.Y.; Kim, J.; Park, H.J.; Kim, E.C.; Park, K.K. Buddlejasaponin IV induces cell cycle arrest at G2/M phase and apoptosis in immortalized human oral keratinocytes. Phytother. Res. 2011, 25, 1503-1510. [CrossRef] [PubMed]

9. Mysak, J.; Podzimek, S.; Sommerova, P.; Lyuya-Mi, Y.; Bartova, J.; Janatova, T.; Prochazkova, J.; Duskova, J. Porphyromonas gingivalis: Major Periodontopathic Pathogen Overview. J. Immunol. Res. 2014, 2014, 476068. [CrossRef] [PubMed]

10. Nakanishi, M.; Rosenberg, D.W. Multifaceted roles of PGE2 in inflammation and cancer. Semin. Immunopathol. 2013, 35, 123-137. [CrossRef] [PubMed]

11. Reddy, N.R.; Kumar, P.M.; Reddy, V.S.; Kumar, A.K.; Babu, M.; Chavan, C.V. Estimation of prostaglandin E2levels in gingival crevicular fluid in periodontal health, disease and after treatment. Contemp. Clin. Dent. 2013, 4, 303-306. [CrossRef] [PubMed]

12. Zhang, J.-M.; An, J. Cytokines, Inflammation, and Pain. Int. Anesthesiol. Clin. 2007, 45, 27-37. [CrossRef] [PubMed]

13. Hienz, S.A.; Paliwal, S.; Ivanovski, S. Mechanisms of Bone Resorption in Periodontitis. J. Immunol. Res. 2015, $2015,615486$. [CrossRef] 
14. Nazir, M.A. Prevalence of periodontal disease, its association with systemic diseases and prevention. Int. J. Health Sci. 2017, 11, $72-80$.

15. Ioannidou, E.; Swede, H.; Fares, G.; Himmelfarb, J. Tooth loss strongly associates with malnutrition in chronic kidney disease. J. Periodontol. 2014, 85, 899-907. [CrossRef]

16. Ara, T.; Nakatani, S.; Kobata, K.; Sogawa, N.; Sogawa, C. The Biological Efficacy of Natural Products against Acute and Chronic Inflammatory Diseases in the Oral Region. Medicines 2018, 5, 122. [CrossRef]

17. Bedran, T.B.L.; Morin, M.P.; Spolidorio, D.P.; Grenier, D. Black tea extract and its theaflavin derivatives inhibit the growth of periodontopathogens and modulate interleukin-8 and beta-defensin secretion in oral epithelial cells. PLoS ONE 2015, 10, e0143158.

18. Hosokawa, Y.; Hosokawa, I.; Ozaki, K.; Nakanishi, T.; Nakae, H.; Matsuo, T. Tea polyphenols inhibit IL-6 production in tumor necrosis factor superfamily 14-stimulated human gingival fibroblasts. Mol. Nutr. Food Res. 2010, 54, S151-S158. [CrossRef]

19. Guimaraes, M.; Coimbra, L.S.; de Aquino, S.; Spolidorio, L.C.; Kirkwood, K.; Rossa, C. Potent anti-inflammatory effects of systemically administered curcumin modulate periodontal disease in vivo. J. Periodontal Res. 2011, 46, 269-279. [CrossRef] [PubMed]

20. Guimaraes, M.; de Aquino, S.; Coimbra, L.S.; Spolidorio, L.C.; Kirkwood, K.; Rossa, J.C. Curcumin modulates the immune response associated with LPS-induced periodontal disease in rats. Innate Immun. 2011, 18, 155-163. [CrossRef] [PubMed]

21. Wang, H.H.; Lee, H.M.; Raja, V.; Hou, W.; Iacono, V.J.; Scaduto, J.; Johnson, F.; Golub, L.M.; Gu, Y. Enhanced efficacy of chemically modified curcumin in experimental periodontitis: Systemic implications. J. Exp. Pharmacol. 2019, 11, 1-14. [CrossRef] [PubMed]

22. Saglam, M.; Köseoğlu, S.; Hatipoglu, M.; Esen, H.H.; Köksal, E. Effect of sumac extract on serum oxidative status, RANKL/OPG system and alveolar bone loss in experimental periodontitis in rats. J. Appl. Oral Sci. 2015, 23, 33-41. [CrossRef] [PubMed]

23. Watanabe, K.; Hirata, M.; Grundler, F.M.W.; Inada, M.; Miyaura, C. Effects of O-methylated (-)-epigallocatechin gallate (EGCG) on LPS-induced osteoclastogenesis, bone resorption, and alveolar bone loss in mice. FEBS Open Bio 2017, 7, $1972-1981$.

24. Wu, Y.-H.; Kuraji, R.; Taya, Y.; Ito, H.; Numabe, Y. Effects of theaflavins on tissue inflammation and bone resorption on experimental periodontitis in rats. J. Periodontal Res. 2018, 53, 1009-1019. [CrossRef] [PubMed]

25. Shetty, S.; Thomas, B.; Shetty, V.; Bhandary, R.; Shetty, R.M. An in-vitro evaluation of the efficacy of garlic extract as an antimicrobial agent on periodontal pathogens: A microbiological study. Arch. Oral. Biol. 2013, 34, 445-451. [CrossRef] [PubMed]

26. Bakri, I.; Douglas, C. Inhibitory effect of garlic extract on oral bacteria. Arch. Oral Biol. 2005, 50, 645-651. [CrossRef] [PubMed]

27. Bachrach, G.; Jamil, A.; Naor, R.; Tal, G.; Ludmer, Z.; Steinberg, D. Garlic Allicin as a Potential Agent for Controlling Oral Pathogens. J. Med. Food 2011, 14, 1338-1343. [CrossRef]

28. Velliyagounder, K.; Ganeshnarayan, K.; Velusamy, S.K.; Fine, D.H. In VitroEfficacy of Diallyl Sulfides against the Periodontopathogen Aggregatibacter actinomycetemcomitans. Antimicrob. Agents Chemother. 2012, 56, 2397-2407. [CrossRef]

29. Giannobile, W.V. Host-Response Therapeutics for Periodontal Diseases. J. Periodontol. 2008, 79, 1592-1600. [CrossRef]

30. Ohshiba, T.; Miyaura, C.; Inada, M.; Ito, A. Role of RANKL-induced osteoclast formation and MMP-dependent matrix degradation in bone destruction by breast cancer metastasis. Br. J. Cancer 2003, 88, 1318-1326. [CrossRef]

31. Lerner, U.H. Stimulation of bone resorption by the kallikrein-kinin system and the coagulation cascade. Acta Orthop. Scand. 1995, 66, 45-50. [CrossRef]

32. Fasanya, H.O.; Siemann, D.W. The Role of Cathepsins in the Growth of Primary and Secondary Neoplasia in the Bone. Osteology 2020, 1, 3-28. [CrossRef]

33. Kusano, K.; Miyaura, C.; Inada, M.; Tamura, T.; Ito, A.; Nagase, H.; Kamoi, K.; Suda, T. Regulation of Matrix Metalloproteinases (MMP-2, -3, -9, and -13) by Interleukin-1 and Interleukin-6 in Mouse Calvaria: Association of MMP Induction with Bone Resorption. Endocrinology 1998, 139, 1338-1345. [CrossRef]

34. Lynch, C.C.; Hikosaka, A.; Acuff, H.B.; Martin, M.D.; Kawai, N.; Singh, R.K.; Vargo-Gogola, T.C.; Begtrup, J.L.; Peterson, T.; Fingleton, B.; et al. MMP-7 promotes prostate cancer-induced osteolysis via the solubilization of RANKL. Cancer Cell 2005, 7, 485-496. [CrossRef] [PubMed]

35. Luo, L.Y.; Katsaros, D.; Scorilas, A.; Fracchioli, S.; Piccinno, R.; Longrais, I.A.R.D.L.; Howarth, D.J.; Diamandis, E. Prognostic value of human kallikrein 10 expression in epithelial ovarian carcinoma. Clin. Cancer Res. 2001, 7, 2372-2379.

36. Yasuda, Y.; Li, Z.; Greenbaum, D.; Bogyo, M.; Weber, E.; Brömme, D.; Humphries, J.E.; Kimber, M.J.; Barton, Y.-W.; Hsu, W.; et al. Cathepsin V, a Novel and Potent Elastolytic Activity Expressed in Activated Macrophages. J. Biol. Chem. 2004, 279, 36761-36770. [CrossRef]

37. Itoh, K.; Udagawa, N.; Katagiri, T.; Iemura, S.; Ueno, N.; Yasuda, H.; Higashio, K.; Quinn, J.M.; Gillespie, M.T.; Martin, T.J.; et al. Bone morphogenetic protein 2 stimulates osteoclast differentiation and survival supported by receptor activator of nuclear factor-kappaB ligand. Endocrinology 2001, 142, 3656-3662. [CrossRef] [PubMed] 